\title{
DESCRIPTION DES ÉTATS ANNUELS ET DES ÉVOLUTIONS DE LA COUVERTURE VÉGÉTALE OBSERVÉE PAR DES SÉRIES TEMPORELLES D'IMAGES MODIS DANS LE PARC NATIONAL DE HWANGE (ZIMBABWE)
}

\author{
Elodie Buard ${ }^{1}$ \\ 1: IGN, COGIT, 73 avenue de Paris, 94160 Saint Mandé, France; Université Paris-Est Marne la Vallée
}

\begin{abstract}
Résumé
Dans le parc de Hwange au Zimbabwe, les grands troupeaux d'herbivores, comme les éléphants, zèbres ou buffles, sont concentrés sur certaines zones. Ces zones subissent donc une forte pression animale. Nous cherchons à étudier l'évolution de la végétation sur ces zones, et plus généralement sur l'ensemble du parc, pour ensuite étudier les liens entre les pratiques spatiales des troupeaux d'animaux et l'évolution de la végétation.

Dans ce papier, nous proposons une démarche pour identifier les évolutions de la couverture végétale, mesurée par le biais des pixels des images satellites MODIS, disponibles à une fréquence mensuelle. L'ensemble de ces images constitue des séries temporelles d'images. Notre démarche permet d'estimer l'importance et le sens de l'évolution de la couverture végétale entre 2003 et 2010 puis d'identifier les lieux d'évolutions.

Chaque image permet d'obtenir des valeurs d'indice NDVI par pixel de $250 \mathrm{~m}$, qui décrivent la biomasse produite. Puis grâce aux séries temporelles de ces images, une analyse évolutive des valeurs NDVI est effectuée. Dans une première partie, nous établissons des profils types de phénologie des végétaux, qui décrivent l'évolution de la biomasse produite par les végétaux au cours de l'année. Comme la phénologie est liée à la pluviométrie, les profils types d'évolution annuelle de la biomasse sont définis pour une année sèche et pour une année humide. Dans une seconde partie, nous nous concentrons sur des évolutions sur une dizaine d'années. Grâce à ces profils types, nous identifions les pixels présentant une faible couverture végétale annuelle en utilisant des seuils d'anormalité statistique. Sur plusieurs années, nous pouvons en dégager des pixels, et donc des lieux du parc, où la couverture végétale a évolué, que ce soit en croissance ou en dégradation.
\end{abstract}

Mots clés : MODIS, couverture végétale, pression animale, NDVI, phénologie, séries temporelles.

\begin{abstract}
In the Hwange National Park in Zimbabwe, herds of herbivores, such as elephants, zebras or buffaloes, are concentrated at certain places. These places undergo a strong animal pressure. We try to study vegetation evolutions on these particular places, but also on the whole park. Then it will enable to study spatial links between spatial practices of animal populations and vegetation evolution.

In this paper, we propose an approach to identify vegetation cover evolutions, measured in pixels of MODIS satellite images. The monthly images constitute time-series MODIS data. Our approach enables to estimate if vegetation cover evolutions are significant between 2003 and 2010, the direction of these evolutions (either negative or positive) and then to identify the places of evolutions.

First, from each image we extract NDVI values to describe produced biomass in each pixel of $250 \mathrm{~m}$. Then we undertake an analysis of NDVI values of each pixel, corresponding to different months in MODIS time-series. In a first part, we build models of crop phenology. It describes evolution of biomass produced yearly by vegetation. Crop phenology is strongly related to the rain falls in the area that is why models are defined for a dry year and for a wet year. In a second part, we focus on vegetation evolutions over a decade. Thanks to these models, we identify pixels where vegetation cover is poor in using thresholds of statistics abnormality. Over several years, some pixels, meaning some places of the park, reveal vegetation cover evolutions whether it is in growing or in damaging.
\end{abstract}

Keywords : MODIS, vegetation cover, animal pressure, NDVI, phenology, abnormal evolution, time-series.

\section{Introduction}

\subsection{Contexte}

Dans un espace naturel, où il y a peu de populations humaines installées, il existe une dynamique "naturelle", composée d'interactions entre les différentes populations animales et végétales, qui composent l'écosystème (Lindeman, 1942). Ces interactions sont de nature trophique et structurelle : elles concernent le réseau de consommateurs successifs et leurs lieux de vie sont interdépendants (Frontier, 1977). Par exemple, 


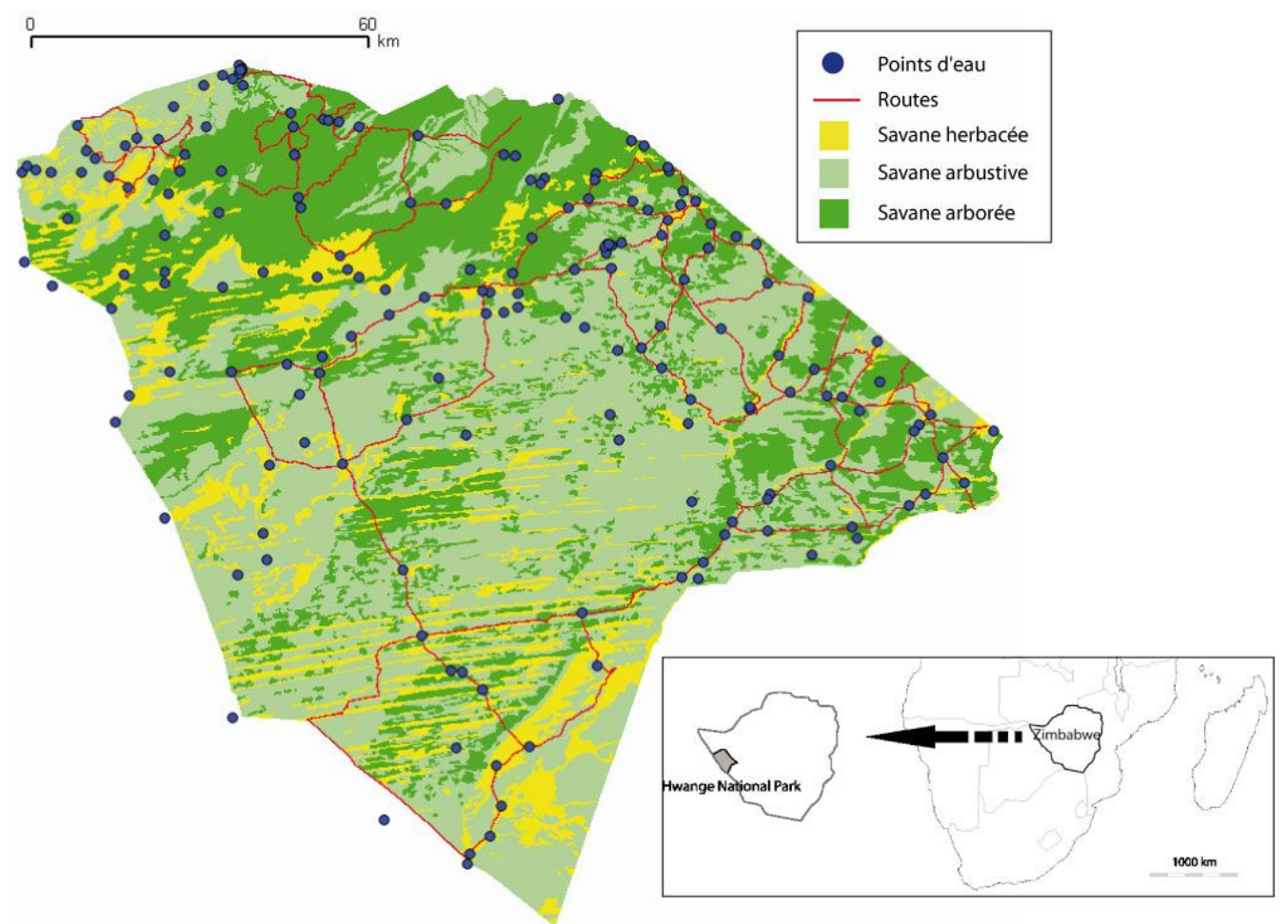

FIGURE 1 : Situation et végétation du parc de Hwange en saison des pluies. Source (Rogers, 2003).

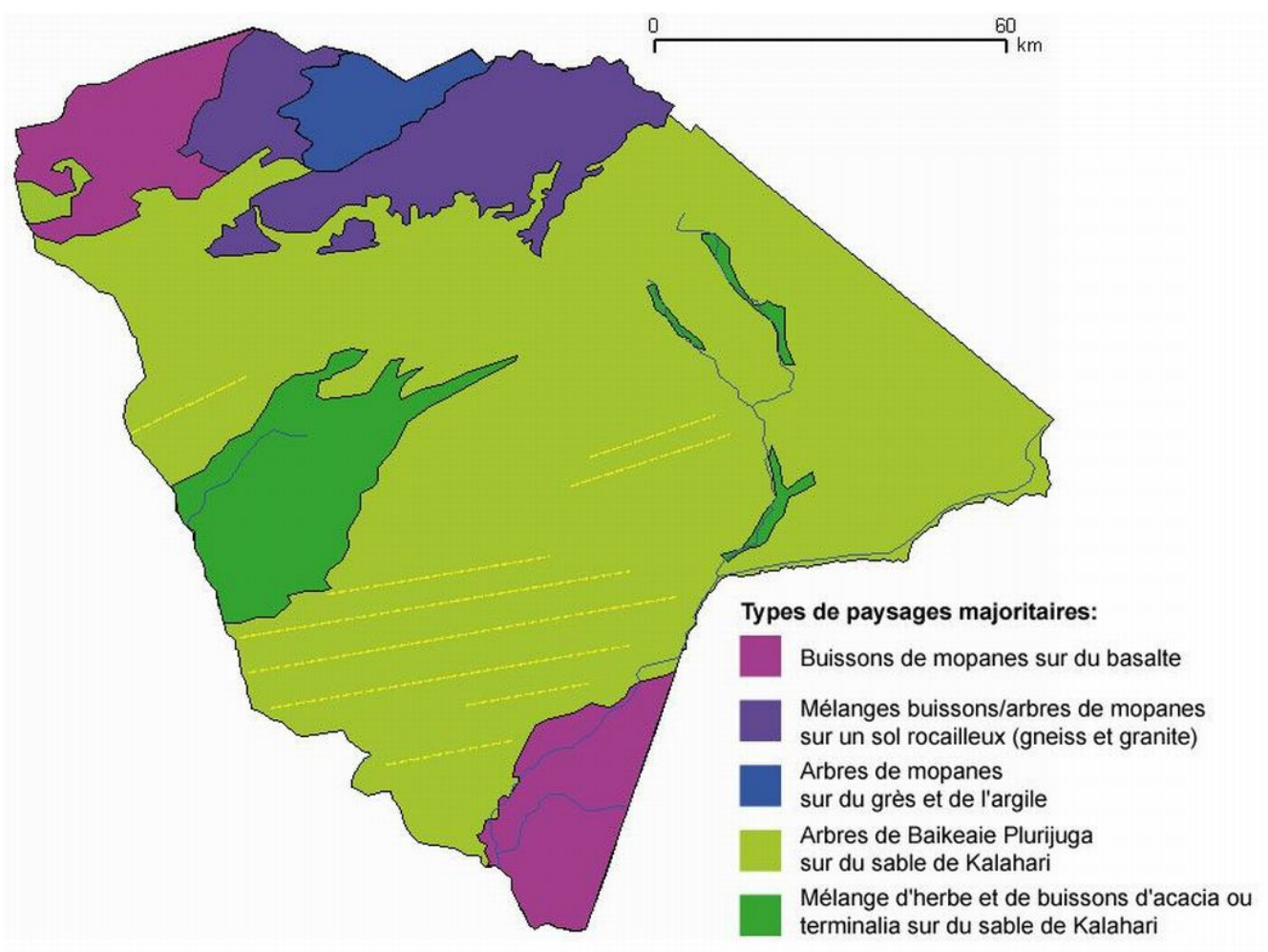

Autres structures caractéristiques du paysage:

— Rivière fossile (vlei)

- - Dune fossile

FIGURE 2 : Carte du paysage du parc de Hwange. 
une population trop nombreuse peut engendrer une diminution des populations dont elle dépend. Ainsi, beaucoup de prédateurs font diminuer les populations d'herbivores; les nombreuses populations d'herbivores dégradent, elles, les ressources végétales, dans leur qualité et quantité (Rackham, 2008). Par ailleurs, les herbivores se localisent dans les lieux où la végétation est la meilleure pour optimiser l'énergie qu'ils en dégagent. Nous nous intéressons dans notre travail à l'action de populations d'herbivores sur leur milieu naturel. Nous nous focalisons donc uniquement sur le système herbivores - végétation - eau de surface, sans prendre en compte ni le sol ni le sous-sol, comme le feraient les agronomes. Les herbivores sont des grands herbivores, définis par un poids dépassant $10 \mathrm{~kg}$ (Lovegrove et Haines, 2004), et en particulier les ongulés sauvages. Ces animaux sont particulièrement intéressants puisqu'ils consomment quotidiennement une grande quantité de végétation et d'eau. Ils sont donc très liés à la végétation et l'eau. Par ailleurs, ces herbivores se déplacent généralement en troupeaux ou en groupe, accentuant par là-même les interactions entre le milieu naturel et les herbivores.

Notre zone d'étude est le parc national de Hwange au Zimbabwe, qui s'étend sur $14600 \mathrm{~km}^{2}$. II s'agit d'une zone atelier de l'Institut Ecologie et Environnement (IEE) du CNRS. La végétation est de type savane, formée d'environ $50 \%$ d'arbustes, $35 \%$ d'arbres et $15 \%$ d'herbe (Valeix et al., 2011). La distinction entre ces types de savane repose sur la hauteur de la végétation : à moins de $30 \mathrm{~cm}$ il s'agit d'herbe, entre $30 \mathrm{~cm}$ et $3 \mathrm{~m}$ d'arbustes et au-delà de $3 \mathrm{~m}$ d'arbres (Rogers, 1993). La savane arborée se trouve majoritairement au Nord du parc, tandis que la savane arbustive se trouve au centre (voir figure 1). La savane herbacée se développe, elle, souvent sur des zones sablonneuses et près des points d'eau.

Le paysage du parc dérive également des espèces végétales et du type de sol. Au centre et à l'est du parc poussent principalement des Baikiaea Plurijuga (il s'agit du teck du Zambèze), des Acacias et des Terminalias où le sol est sablonneux, tandis qu'au nord et au sud se trouvent des Colosphernum Mopane sur des roches basaltiques (voir figure 2).

Le climat y est semi-aride; il comprend deux saisons, une sèche, une dite "des pluies", chacune durant en moyenne 6 mois. Pendant la saison sèche, les végétaux se dessèchent, constituant de faibles ressources pour les animaux, tandis que l'eau est présente ponctuellement sous forme de points d'eau. En saison des pluies, la végétation est très développée et l'eau est abondante (apparition de rivières). Une autre caractéristique du parc de Hwange est qu'il abrite un grand nombre d'espèces animales qui sont protégées par des lois en raison de leur rareté à l'échelle internationale, comme les lions, zèbres, buffles ou éléphants (UICN, 2008). Leurs populations ne sont donc pas régulées par les populations humaines.

Dans cette zone, les écologues ont constaté une évolution des populations animales présentes. Les populations d'éléphants sont de plus en plus nombreuses et atteignent une des plus fortes densités au monde, allant jusqu'à 5 individus par $\mathrm{km}^{2}$ (Chamaillé-Jammes et al., 2009; Van Aarde et Jackson, 2006), ce qui est un paradoxe compte tenu de leur rareté à l'échelle internationale. Les populations sont en fait concentrées sur certaines zones en saison sèche, comme les points d'eau ou les zones où il existe encore de la végétation verte (voir figure 3).

Cette concentration d'herbivores implique que les

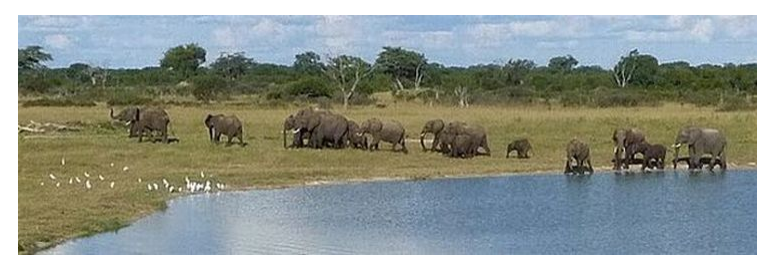

FIGURE 3 : Groupe d'éléphant à un point d'eau.

végétaux sont consommés de manière non uniforme, certaines zones étant rasées, d'autres non. Par ailleurs, certaines de ces populations modifient la structure même de la végétation. C'est le cas de l'éléphant, qui casse les branches et déracine les arbres très rapidement (Owen-Smith, 1988), que ce soit pour sa consommation ou à cause de ces passages répétés. Les fortes pressions animales pourraient donc engendrer des fortes hétérogénéités et des fortes modifications dans la végétation.

II s'agit donc d'étudier la question du surpâturage pour des espèces d'herbivores sauvages et protégées. Si les écologues constatent un surpâturage est local à certains moments de l'année, encore faut-il pouvoir valider ce constat en identifiant les zones concernées, à certaines dates, ainsi que le type de végétation en jeu. S'agit-il de zones très accessibles ou ayant une végétation particulièrement appétente pour les herbivores? Par ailleurs, y a-t- il des effets de dégradation de la végétation à l'échelle du parc et sur une longue période due aux populations animales? Ceci implique d'étudier les évolutions de la végétation à différentes échelles spatiales et temporelles.

Ces questionnements thématiques soulèvent des questionnements méthodologiques, centrés sur les méthodes à mettre au point pour mesurer, identifier, évaluer les déplacements d'animaux et l'évolution de leur milieu. Notre méthodologie repose sur une approche spatiale des interactions entre animaux et milieu, par une caractérisation de l'espace selon ces variables : pra- 
tiques de déplacement des populations animales d'une part, et évolution du milieu naturel d'autre part. Puis nous étudions les relations entre ces variables par des statistiques. Notons que dans notre travail nous appréhendons le milieu naturel par l'occupation du sol, couverture physique de la Terre (FAO, 1998), et estimé par des images satellites, puisqu'il n'existe pas de base de données décrivant l'occupation du sol sur notre zone.

\subsection{Objectif de l'article}

Dans ce papier, nous présentons uniquement notre méthodologie et quelques résultats concernant l'identification de l'évolution de l'occupation du sol. Nous considérons donc que l'occupation du sol correspond ici à la couverture végétale, puisque le parc de Hwange est très peu habité et peu aménagé. Les méthodologies mises au point pour identifier et construire les pratiques spatiales des troupeaux d'herbivores sont décrites en détail dans (Buard, 2013).

Ici, nous utilisons des séries temporelles d'images provenant du satellite MODIS (Moderate Resolution Imaging Spectroradiometer). Ce sont des ensembles d'images MODIS, de moyenne résolution spatiale $(250 \mathrm{~m}$ et plus), prises à différentes périodes et régulièrement. L'intérêt du capteur MODIS est sa haute répétitivité temporelle qui permet une visite fréquente des lieux observés. Les images MODIS ont souvent été utilisées pour la présence et l'évolution de l'occupation du sol. Pour citer quelques travaux : évolution de la culture de soja dans la forêt amazonienne (Arvor, 2009), détection de plantations forestières au Chili (Paegelow et al., 2012), évolution de l'activité végétale des parcelles forestières en Bourgogne (Doll, 2010), évolution des feux à Madagascar (Jacquin, 2010) ou évolution des plans d'eau dans le désert tunisien (Najib, 2010). Ces travaux étudient d'autres milieux, mais toujours des milieux naturels ou forestiers. L'intérêt de ce type de capteur est donc la répétitivité temporelle pour étudier les évolutions. Un capteur satellite plus classique comme Landsat ne permettait pas de couvrir la zone d'étude de manière exhaustive (problèmes de nuages sur la zone en particulier lors de la saison des pluies) et régulière.

Dans ces travaux, ce sont les indices décrivant la végétation ou leurs évolutions au cours du temps qui sont analysés. D'ailleurs, les images MODIS fournissent directement douze indices de végétation qui mesurent l'état de la couverture végétale dans les pixels. Ces indices diffèrent dans leur prise en compte des réflectances mesurées dans les longueurs d'ondes observées.

L'étude des indices fournis par les images MODIS met en évidence les évolutions saisonnières de la couverture végétale des pixels (appelée la phénologie), ainsi que les évolutions sur une plus longue période de la couverture végétale.

Le but de notre méthodologie est d'obtenir les lieux où la couverture végétale a beaucoup évolué et d'estimer les modalités de cette évolution. Nous nous sommes particulièrement concentrés sur les dégradations de la couverture végétale, ce qui est lié à notre problématique. En effet, notre hypothèse initiale est que les troupeaux d'herbivores avaient un effet négatif, et non positif, sur la végétation. Cependant, la méthodologie est générale et pourrait s'appliquer (à condition de changer les valeurs des seuils de détection des évolutions) à d'autres problématiques liés à l'évolution de la couverture végétale et qui pourraient être centrés sur une croissance de celleci, et non une dégradation.

Tout d'abord, en section 2, nous donnons des détails sur ces images. En section 3, nous présentons notre méthode pour décrire la phénologie de la végétation et identifier des profils types d'évolution annuelle de la végétation. Enfin, en section 4, nous identifions les pixels décrivant une évolution de la couverture végétale pendant une dizaine d'années.

\section{Données disponibles}

Nous voulons utiliser des séries temporelles d'images du satellite MODIS mesurant directement des indices de végétation. Or le choix des images est complexe car il existe une grande variété de produits MODIS. Nous nous focalisons sur les images permettant de suivre l'occupation du sol terrestre. Elles ont un préfixe "MOD", les autres images permettant de suivre l'atmosphère ou les océans. Dans les images d'occupation du sol MODIS, nous choisissons d'extraire les images mesurant directement des indices de végétation. II s'agit de la gamme d'images "MOD13". Enfin, dans cette gamme, six types d'images existent, variant en résolution spatiale (de $250 \mathrm{~m}$ à $1 \mathrm{~km}$ ) et en répétitivité temporelle (de 16 jours à 1 mois). Ces types d'images sont dénommés par des lettres et des chiffres : $A 1, A 2$, $A 3, C 1, C 2$ et $Q 1$. Finalement, nous utilisons les images MOD13Q1 qui sont les plus précises spatialement et temporellement : elles correspondent à une résolution de $250 \mathrm{~m}$ et une répétitivité de 16 jours.

Sur ces 16 jours, les indices de végétation sont agrégés pour ne fournir qu'une seule image représentative. La méthode d'agrégation employée dépend du nombre de mesures journalières exploitables (Huete et al., 2002) : l'observation des pixels peut être altérée à cause des nuages et de certains angles de prise de vue. Une image MODIS correspond donc toujours à une image composite issue d'agrégations temporelles, pixels par pixels. Les différentes méthodes d'agrégations sont décrites dans (Arvor, 2009). Dans le cas le plus général, on calcule la moyenne de l'indice de végétation de cinq jours d'observation.

En pratique, il existe des périodes de 16 jours pour lesquelles l'image MODIS est indisponible, en particulier pendant les saisons des pluies. Nous ne pouvons obtenir qu'une image par mois dans cette saison. Pour garantir une régularité des sources de données, nous 
nous contraignons à n'utiliser qu'une image MOD13Q1 par mois.

Les corrections radiométriques à appliquer sur les images sont effectuées avant livraison.

Douze indices sont calculés et peuvent être extraits par pixels, entre autres : NDVI (Normalized Difference Vegetation Index), EVI (Enhanced Vegetation Index), les réflectances dans le bleu, rouge, proche infrarouge, moyen infrarouge, l'angle du soleil ou la qualité des observations pixellaires. Les deux indices composites, intégrant des réflectances dans différentes longueurs d'ondes, sont le NDVI et l'EVI. Ils mesurent tous les deux une importance de l'activité végétale, soit une importance de la biomasse. L'indice NDVI est un des plus utilisés pour étudier la végétation. II retient notre attention particulièrement parce qu'il est adapté aux régions semi-arides, comme le parc de Hwange, où la végétation n'est pas trop dense (Richard et Poccard, 1998). Au contraire, l'indice EVI est utilisé pour l'étude de la végétation tropicale (Huete et al. 2002), parce que NDVI sature lorsque la végétation est très active.

L'indice NDVI se calcule comme suit (Rouse et al., 1974) :

$$
N D V I=\frac{P I R-R}{P I R+R} .
$$

Ses valeurs varient entre -1 et +1 . Une forte valeur correspond à une couverture végétale dense dans le pixel; une faible valeur correspond à du sol nu ou de la végétation sèche. Les valeurs peuvent ensuite être classifiées pour recréer l'occupation du sol (Ferreira et al., 2003). A titre d'exemple, la figure 4 présente une classification des valeurs prises par l'indice NDVI sur le parc de Hwange, dans les pixels d'une image MODIS datée du 29 août 2010 (correspondant à la date initiale de la période de 16 jours), donc en saison sèche. Les valeurs NDVI varient dans le temps, puisque la production de feuillage et d'herbe est plus ou moins intense dans l'année. Dans cet exemple, la végétation est sèche.

Les seuils de cette classification ont été fixés en s'appuyant sur les valeurs clés trouvées dans la littérature (Derrien et al., 1992 ; Arvor et Dubreuil, 2006) :

- Si valeur NDVI du pixel $<0,25$, alors le sol est nu ou les végétaux desséchés dans ce pixel;

- Si 0,25< valeur NDVI du pixel $<0,4$, alors les végétaux sont en début ou en fin de développement dans ce pixel ;

- Si 0,4 < valeur NDVI du pixel < 0,55, alors les végétaux sont au milieu ou au maximum de leur développement dans ce pixel.

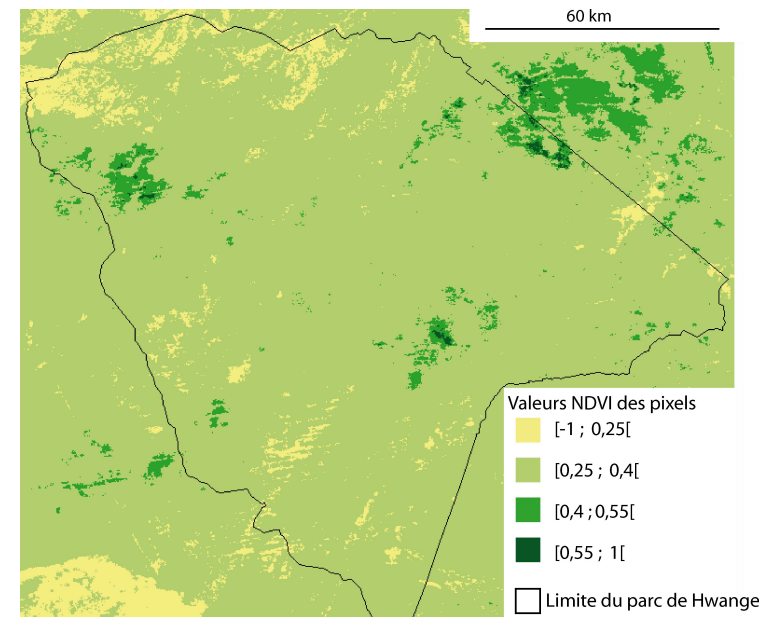

FIGURE 4 : Image MODIS de type MOD13Q1 datée du 29 août 2010 avec classification des valeurs de l'indice NDVI.

\section{Description de la phénologie des végétaux par des séries temporelles d'images MODIS}

Dans cette section, nous analysons l'évolution des valeurs NDVI des pixels. Cette évolution se déroule sur plusieurs temporalités. D'abord, nous étudions les valeurs NDVI mensuelles des végétaux qui évoluent au cours de l'année (en 3.1). Dans ce cas il s'agit de phénologie. Puis, nous étudions l'évolution sur une dizaine année des valeurs NDVI annuelles, calculées par agrégation des valeurs NDVI mensuelles (en 3.2). Cette analyse permet d'avoir une idée de l'évolution de la végétation sur une longue période.

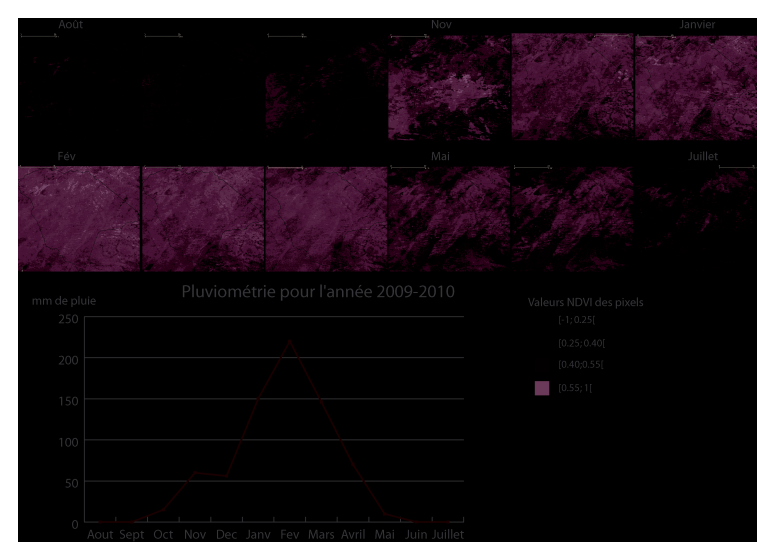

FIGURE 5 : Aperçu des variations annuelles des valeurs NDVI des pixels. Chaque imagette observe le parc s'étendant sur une zone de $140 \mathrm{~km}$ * $100 \mathrm{~km}$ à un mois donné, variant entre septembre 2009 et août 2010 .

\subsection{Analyse des valeurs NDVI mensuelles au cours de l'année}

Les valeurs NDVI qui dépendent de la biomasse, reflètent donc aussi les phases de développement 

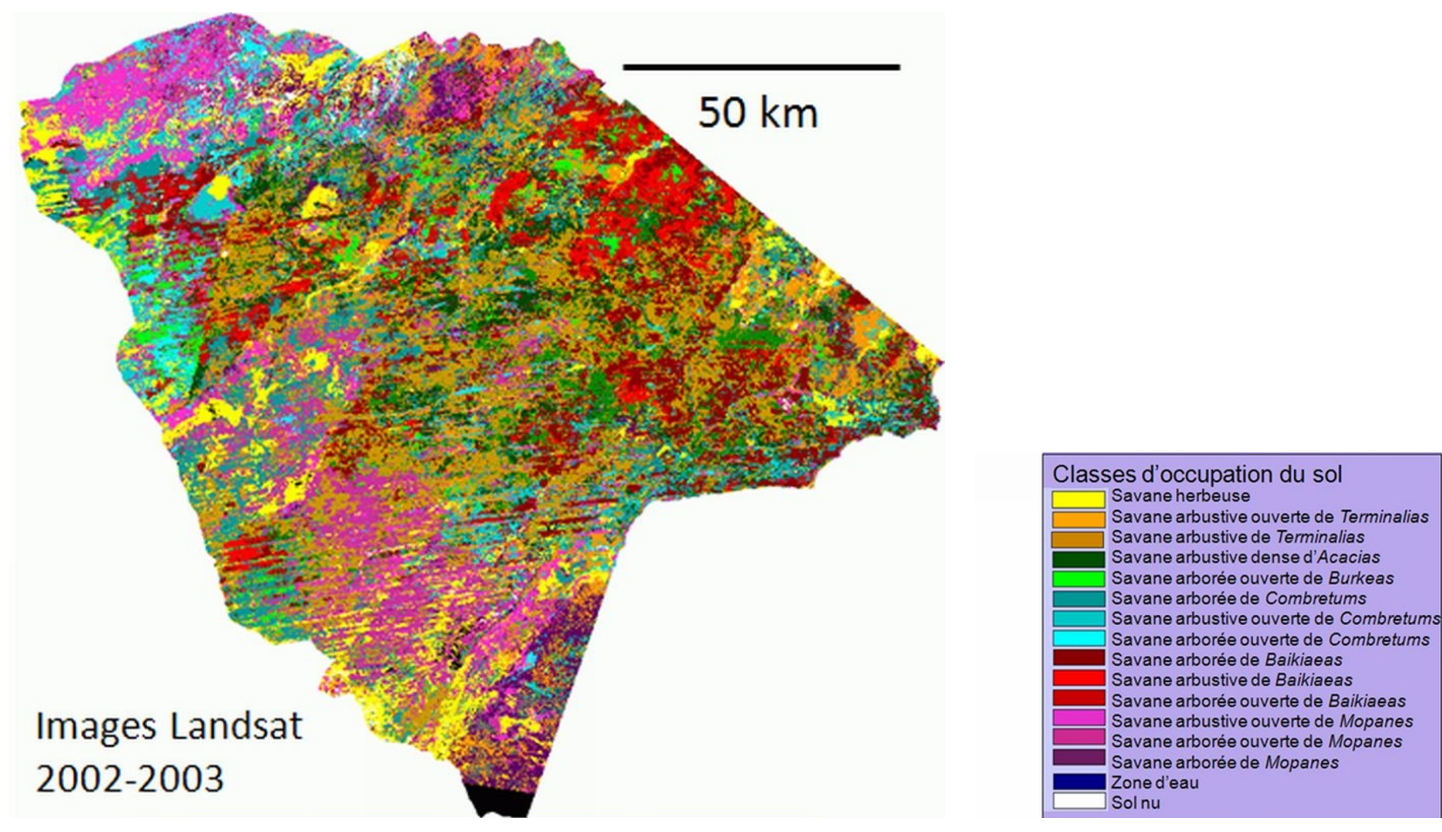

FIGURE 6 : Image Landsat classifiée issue de deux images brutes Landsat 2002-2003.

de la végétation, ce qu'on appelle phénologie : elles sont maximales lorsque la végétation est développée, c'est-à-dire ici en mars, et minimales lorsqu'elle est sèche, ici en août. Pour décrire finement la phénologie, il faut connaître les dates clés de croissance et sénescence, et les valeurs NDVI maximales et minimales. La phénologie nous permettra ensuite d'identifier des profils types d'évolution annuelle de la couverture végétale en identifiant des seuillages pertinents des indices NDVI.

Pour étudier les cycles phénologiques, nous considérons un cycle pluviométrique, allant de début septembre de l'année n-1 à août de l'année n. Nous illustrons cette étude par 12 images prises entre 2009 et 2010, en utilisant la même classification que précédemment, en figure 5. Cette cartographie multi temporelle permet d'estimer visuellement les variations des valeurs NDVI sur l'ensemble du parc, qui mettent bien en évidence les deux saisons. Un parallèle est fait entre la pluviométrie et les valeurs NDVI sur l'ensemble du parc. Ce lien pluviométrie - NDVI a été démontré dans (Marteau, 2006). Néanmoins, localement, certaines zones peuvent quand même produire de la biomasse, même en saison sèche.

Maintenant, notre tâche est de décrire en détails la phénologie des végétaux dans le parc. Pour cela, il faut étudier l'évolution annuelle des valeurs NDVI de chaque type d'espèce végétale.

De manière générale, peu de cartes de végétation existent sur la zone, hormis la carte établie par (Rogers, 2003) présentée en figure 1. II est également difficile et long de collecter des données de télédétection et de les valider sur le terrain.
Une seule image provenant des travaux de (Ganzin et al., 2008) existe. II s'agit d'une image Landsat classifiée, de résolution $30 \mathrm{~m}$. La classification est supervisée, s'appuyant sur des points terrain, et combine des images brutes Landsat de deux saisons en 2002-2003. Ainsi cette image classifiée est indépendante de la saison considérée. Il s'agissait en 2008 d'établir une carte de végétation (voir figure 6), plus précise que. La précision globale de l'algorithme de classification (distance minimale entre les signatures spectrales des pixels et les classes d'occupation du sol prédéfinies) est de $68 \%$ et l'indicateur Kappa de 0,52. La classification est donc valide, mais de faible qualité (indice Kappa < 0,6, cf. Hubert-Moy et al., 2001).

Cette image est la plus récente référençant la végétation et étant validée par des travaux de terrain. C'est pourquoi, même si elle est datée de 2002-2003 (seulement), nous extrapolons les espèces végétales présentes en 2002-2003 et en 2009-2010.

Dans l'idéal, nous devrions travailler sur la résolution de $30 \mathrm{~m}$, qui est la plus précise pour identifier les espèces végétales présentes. Cependant, nous cherchons à caractériser les images MODIS par cette image Landsat classifiée. Ainsi, nous attribuons à chaque pixel de MODIS, de taille $250 \mathrm{~m} \times 250 \mathrm{~m}$, l'espèce végétale qui est majoritaire en surface d'après l'image Landsat, rééchantillonnée. Nous considérons ainsi qu'à un ensemble de pixels MODIS correspond un seul type de couverture végétale, qui serait une moyenne sur $250 \mathrm{~m} \times 250 \mathrm{~m}$.

La seconde étape consiste à effectuer les moyennes par mois des valeurs NDVI des pixels de chaque type de couverture végétale, ce qui permet d'obtenir des 
types phénologiques moyens par type de couverture végétale. Pour simplifier, nous considérons ici trois types de couvertures végétales: l'herbe, les arbustes et les arbres. Rappelons que l'herbe couvre $15 \%$ du parc, les arbustes $35 \%$ et les arbres $50 \%$. Globalement, ces trois strates de végétation se comportent de la même façon dans leur développement végétal (voir figure 7) : les valeurs NDVI sont maximales en mars $(0,6)$ et minimales en août $(0,3)$. La durée de croissance est donc de 7 mois ; celle de senescence de 5 mois.

Dans chaque strate et chaque mois, les valeurs NDVI peuvent varier de $\pm 0,5$, ce qui est visible grâce aux barres d'erreur du graphique. Ainsi, même si les valeurs NDVI des herbes semblent se situer légèrement sous celles des arbustes et des arbres, cette différence est à relativiser puisqu'il existe des variations intra-strates.

Evolutions NDVI 2009-2010

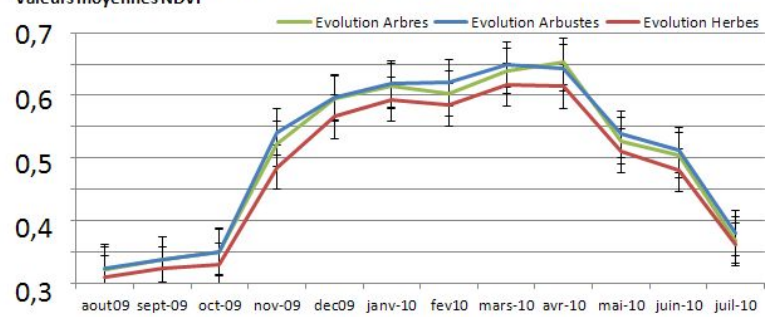

FIGURE 7 : Evolutions annuelles des valeurs NDVI pour l'année 2009-2010 et pour les strates d'arbres (en vert), d'arbustes (en bleu) et d'herbes (en rouge).

Notons que l'année 2009-2010 est une année pluvieuse. Si on crée le même graphique pour d'autres années, on remarque que pour les années sèches, les valeurs NDVI sont plus faibles (de l'ordre de 0,5), ce qui est logique puisque la végétation devrait être moins développée. Les valeurs NDVI maximales et minimales sont donc directement liées à la pluviométrie. Par ailleurs, les valeurs maximales sont atteintes plus tôt dans l'année en année sèche : en janvier au lieu de mars. Ceci révèle aussi une différence dans la durée des pluies : en année sèche, les pluies durent moins longtemps.

Enfin, si on regarde de plus près le comportement de chaque espèce d'arbres et d'arbustes, on voit que les espèces d'arbres renvoient pratiquement les mêmes valeurs NDVI, ce qui n'est pas le cas pour les arbustes: l'acacia a des valeurs NDVI supérieures aux autres arbustes. Cette différence des comportements d'arbustes est amplifiée pour les années sèches, où l'acacia conserve de fortes valeurs NDVI. Ceci met en évidence une certaine résistance à la sécheresse.

Ces profils types nous serviront de base par la suite. Pour résumer, nous considérons trois profils de couverture végétale :
- L'herbe;

- Les arbres/arbustes, qui ont des valeurs NDVI supérieures à celles de l'herbe, et ce quelle que soit la pluviométrie;

- Les arbustes résistants à la sécheresse, dont les valeurs NDVI sont élevées, particulièrement en année sèche.

\subsection{Evolution des valeurs NDVI annuelles sur 10 ans}

Une méthode pour donner une tendance de l'évolution à plus long terme de la végétation est d'étudier des valeurs NDVI annuelles et non mensuelles pour se soustraire aux variations dues à la phénologie des végétaux.

Jacquin (2010) a proposé un indice pour caractériser la dynamique des savanes qui repose sur la somme des valeurs NDVI mensuelles. On agrège ainsi les 12 valeurs NDVI mensuelles de chaque pixel pour obtenir une valeur NDVI annuelle par pixel et on calcule ensuite une valeur NDVI annuelle moyenne, sur l'ensemble du parc. L'agrégation est donc temporelle (l'année) et spatiale (le parc). Selon (Jacquin, 2010), cet indice permet d'évaluer la biomasse cumulée produite par an. L'intérêt de cet indice est d'étudier son évolution sur plusieurs années, en fonction des images disponibles. De façon théorique, cet indice varie entre -12 et +12 .

La figure 8 présente les indices NDVI annuels moyens obtenus sur le parc, par des séries temporelles d'images MODIS entre 2000 et 2010 . Mis en parallèle avec le graphique de pluviométrie annuel, on distingue les ressemblances entre les deux courbes, qui évoluent dans le même sens. Le graphique des indices NDVI révèle des différences dans les indices, entre les années sèches (valeurs basses, entre 4 et 5 ) et les années humides (valeurs plutôt hautes, entre 5 et 6 ).

$\mathrm{Ce}$ qui est intéressant dans ce graphique c'est la pente positive de cette courbe, de coefficient directeur 0,13 . Ceci signifie que la biomasse est en évolution progressive dans le parc. C'est un premier résultat qui montrerait que la couverture végétale ne se dégrade pas au fil des années, et au contraire est en croissance.

Pour résumer cette partie, les valeurs NDI mensuelles des pixels nous ont permis de construire des profils types d'évolution annuelle de la végétation. Puis une agrégation de ces valeurs a permis d'estimer l'évolution décennale de la végétation sur le parc de Hwange.

\section{Identification de pixels présentant des états anormaux de la couverture végétale et leurs évolutions sur 10 ans}

L'indice NDVI permet d'identifier la biomasse, et donc aussi les pixels où la végétation se développe de façon anormale, par rapport aux valeurs NDVI attendues si on se réfère aux profils types préétablis. Cette anormalité 


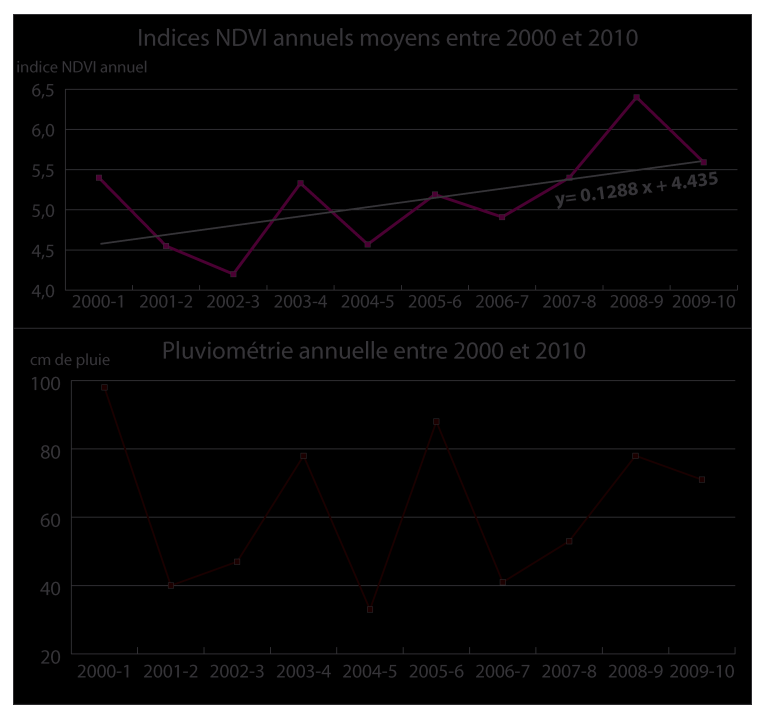

FIGURE 8 : Evolution de l'indice NDVI annuel moyen sur le parc d'après des séries temporelles d'images MODIS et lien avec la pluviométrie annuelle entre 2000 et 2010.

est identifiée par pixel et est estimée à différentes temporalités :

- Mensuelle (valeurs NDVI très hautes ou très basses à un mois donné) ;

- Annuelle (valeurs NDVI annuelles des pixels très hautes ou très basses);

- Sur plusieurs années.

Ces anormalités de croissance de la couverture végétale peuvent être dues à plusieurs facteurs, comme le climat ou des trop fortes pressions sur le sol. Notre intérêt est de repérer les pixels où la couverture végétale se développe trop faiblement. II faut identifier alors des valeurs NDVI en dessous de la valeur attendue par le profil type de la végétation. L'indice NDVI est alors un bon indicateur de végétation dégradée (Meneses-Tovar, 2011).

Statistiquement, le terme d' "anormal" correspond à une valeur plus faible que (moyenne - $2 \times$ écart type). Nous considérons que dans cet intervalle de valeurs, la couverture végétale est très faible. Cependant, nous ajoutons à cette sélection un autre intervalle de valeur : celui dont les valeurs ne sont pas dans le premier intervalle et qui se situent sous (moyenne - 1×écart type). Ici, la couverture végétale est faible. Ces deux intervalles permettront de sélectionner les pixels qui présentent des états dé dégradés de la couverture végétale.

Reste à savoir comment sélectionner ces valeurs moyennes et ces écarts types, puisque les images desquelles ils sont issus varient au cours de l'année.

La figure 9 présente les étapes suivies dans cette section : nous identifions des états annuels de la couverture végétale grâce aux profils types de la végétation et à des pixels de référence (voir 4.1) puis nous étudions l'évolution de la couverture végétale en 7 ans (4.2).

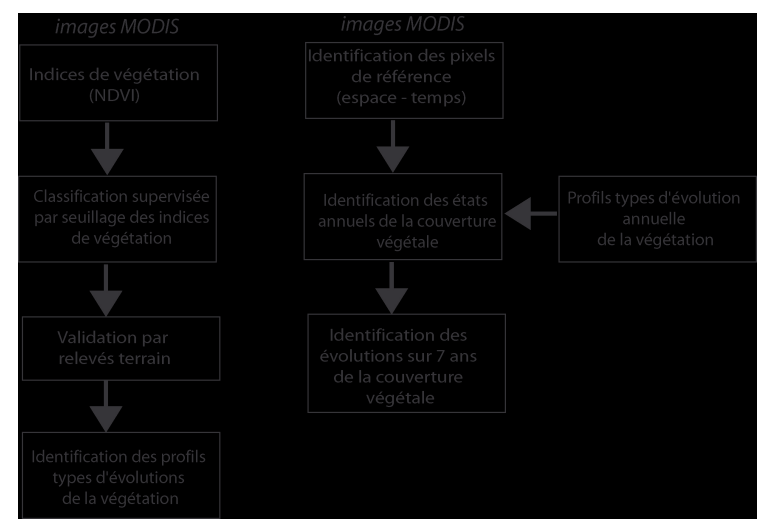

FIGURE 9 : Déroulé de notre méthodologie pour identifier les états annuels et les évolutions de la couverture végétale.

\subsection{Principes d'identification des états annuels de la couverture végétale dans les pixels}

Comme les pixels sont à la fois situés dans l'espace (dans chaque image) et dans le temps (à un certain mois), il existe plusieurs méthodes pour calculer les moyennes et écarts types de référence. Ces deux méthodes sont illustrées sur la figure 10, chaque grille représentant une image prise à un certain mois.

La première méthode (dont l'emprise est matérialisée en vert sur la figure) repose sur une analyse de l'ensemble de l'image: les valeurs moyennes et les écarts types sont identifiés par image, ce qui est équivalent à un mois donné. Ces valeurs reposent sur les profils types identifiés précédemment et établis pour différentes espèces végétales. Par cette méthode, les pixels dont la valeur NDVI est à inférieure (moyenne - écart type) ou (moyenne - $2 \times$ écart type) sont considérés comme présentant des écarts spatiaux, sous-entendu : par rapport aux valeurs NDVI des autres pixels de l'image. Ces pixels sont représentés par des cellules colorées en vert dans la figure. On qualifie cette méthode de spatiale.

La seconde méthode (emprise en rose) considère chaque zone de $250 \mathrm{~m} \times 250 \mathrm{~m}$, soit la taille d'un pixel. Cette zone est observée par une série d'images au cours de l'année. Elle correspond à un ensemble de pixels colocalisés, chacun issu d'une image mensuelle. Par ailleurs, ces pixels correspondent à une même espèce végétale, puisque nous considérons que l'espèce végétale n'a pas évolué spatialement en une année, c'est-à-dire ne s'est ni étendue ni réduite. Cette hypothèse a du sens puisque la croissance des végétaux est limitée : d'abord, seuls 6 mois de l'année sont utilisés dans la croissance de la plante dans le parc; puis cette croissance n'atteint jamais annuellement la taille d'un pixel. De la même façon, on peut calculer des valeurs 
et des écarts types à partir des pixels colocalisés. Si certains pixels ont des valeurs NDVI qui se trouvent sous (moyenne - écart type) ou (moyenne - $2 \times$ écart type), alors le pixel présente des écarts temporels par rapport aux valeurs NDVI des autres pixels colocalisés, comme les pixels colorés en rose. On qualifie cette méthode de temporelle.

Ces deux méthodes sont complémentaires : l'une étant horizontale sur l'espace, l'autre verticale sur le temps. Seuls quelques pixels présentent des anormalités d'évolution dans le temps et l'espace, comme le pixel du mois de janvier au milieu de l'image. Ces pixels présentent alors des écarts spatiotemporels. Ce dernier cas est plus restrictif dans les pixels identifiés.

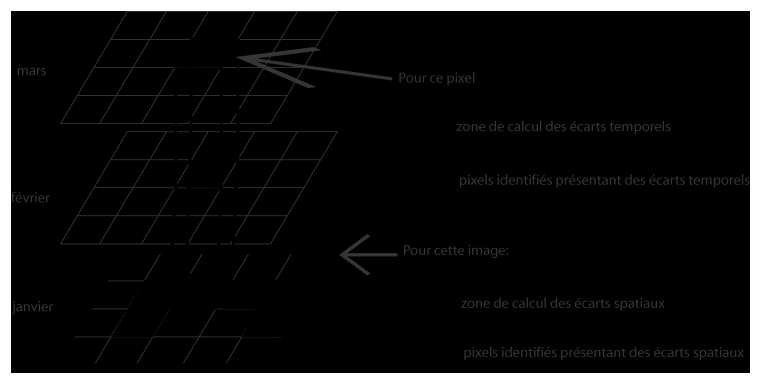

FIGURE 10 : Principe des méthodes spatiales et temporelles pour identifier des pixels dont les valeurs NDVI sont statistiquement anormales.

Dans ce papier, nous détaillons une des deux méthodes présentées : la méthode spatiale. Au sein même de cette méthode, on peut identifier des moyennes et écarts types à temps fixe, en se servant uniquement d'une image, ou à temps cumulé, en considérant un temps agrégé (l'année). La plus intuitive est de considérer un temps fixe. On essaye alors d'identifier où la couverture végétale est la plus faible à un mois donné. Toute la question est de bien choisir le ou les mois considérés. A priori deux mois semblent intéressants :

- celui où la valeur NDVI est maximale : le mois de janvier ou mars selon les types d'années;

- celui où elle est minimale : le mois d'août.

Ces mois reflètent respectivement la quantité de végétation maximale disponible et après des mois de sécheresse.

Pour les mois de janvier/mars et d'août, les moyennes et écarts types des valeurs NDVI peuvent être calculées par strate de végétation (arbres, arbustes et herbe) puisqu'ils n'évoluent pas de la même façon. Ceci permet ensuite d'identifier les pixels se trouvant dans :

- l'intervalle 1, c'est-à-dire que les valeurs NDVI des pixels se situent sous (moyenne - écart type). II s'agit de couverture végétale faible.

- l'intervalle 2 dans lequel les valeurs NDVI des pixels sont inférieures à (moyenne $-2 \times$ écart type). II s'agit de couverture végétale très faible.

\begin{tabular}{|c|c|c|}
\hline & Intervalle 1 & Intervalle 2 \\
\hline \multirow{3}{*}{ Août 2009 } & $\mathrm{H}: 12,7 \%$ & $\mathrm{H}: 0,1 \%$ \\
& $\mathrm{~B}: 14 \%$ & $\mathrm{~B}: 0,7 \%$ \\
& $\mathrm{~A}: 11,8 \%$ & $\mathrm{~A}: 0,23 \%$ \\
\hline \multirow{3}{*}{ Janvier 2010 } & $\mathrm{H}: 17 \%$ & $\mathrm{H}: 1,6 \%$ \\
& $\mathrm{~B}: 15,4 \%$ & $\mathrm{~B}: 2,9 \%$ \\
& $\mathrm{~A}: 14,2 \%$ & $\mathrm{~A}: 3,2 \%$ \\
\hline
\end{tabular}

TABLE 1 : Proportions de pixels inclus dans les intervalles 1 et 2 (correspondants à une couverture végétale faible et très faible), en janvier 2010 et août 2009, pour l'herbe (H), les buissons (B) et les arbres (A).

Les pixels identifiés dans l'un ou l'autre des intervalles peuvent varier. Si on étudie la diversité statistique des pixels se trouvant dans ces deux intervalles, pour janvier 2010 et août 2009 , on obtient le tableau 1.

On remarque que peu de pixels enregistrent une faible couverture végétale : ils représentent moins de $20 \%$ des pixels du parc, quel que soit le mois et la strate de végétation considérés. Par ailleurs, il y a plus de pixels identifiés en janvier qu'en août. Autrement dit l'écart spatial entre les valeurs NDVI des pixels est supérieur en janvier par rapport à août, où tous les végétaux sont secs. La cartographie des pixels de faible couverture végétale au mois d'août 2009 est présentée en figure 11. En blanc figurent les pixels enregistrant une couverture végétale normale. Ils sont largement majoritaires sur le parc. En couleur sont représentés les pixels présentant une faible couverture végétale (intervalle 1 : couleurs claires) ou très faible (intervalle 2 : couleurs foncés). On distingue ici les states de végétation, c'est-à-dire les herbes, arbustes et arbres, puisqu'ils servent à identifier les moyennes et écarts types.

On voit dans cette figure que les pixels de faible couverture végétale sont plutôt situés au nord du parc, et il s'agit d'arbres. Si on fait ce même travail pour le mois de janvier, alors, la zone du parc qui a une faible couverture végétale est, cette fois, le sud, qui est peuplée d'arbustes. Dans le centre du parc, la végétation pousse normalement en 2009-2010.

Ces deux cartes résultantes (janvier et août) peuvent être combinées pour en ressortir les pixels de faible couverture végétale en janvier et août. La méthode temporelle peut ensuite être appliquée en considérant plusieurs mois de l'année pour chaque pixel. Nous ne la détaillons pas ici, mais elle est très similaire à la méthode spatiale : calcul des moyennes et écarts types par pixels puis agrégation à l'ensemble de l'image.

L'étape finale est d'obtenir une carte combinant les deux méthodes précédentes. Pour cela, on calcule la valeur d'écart maximal entre la carte issue de la méthode spatiale sur un an et la carte issue de la méthode temporelle sur un an. Le résultat se trouve en 


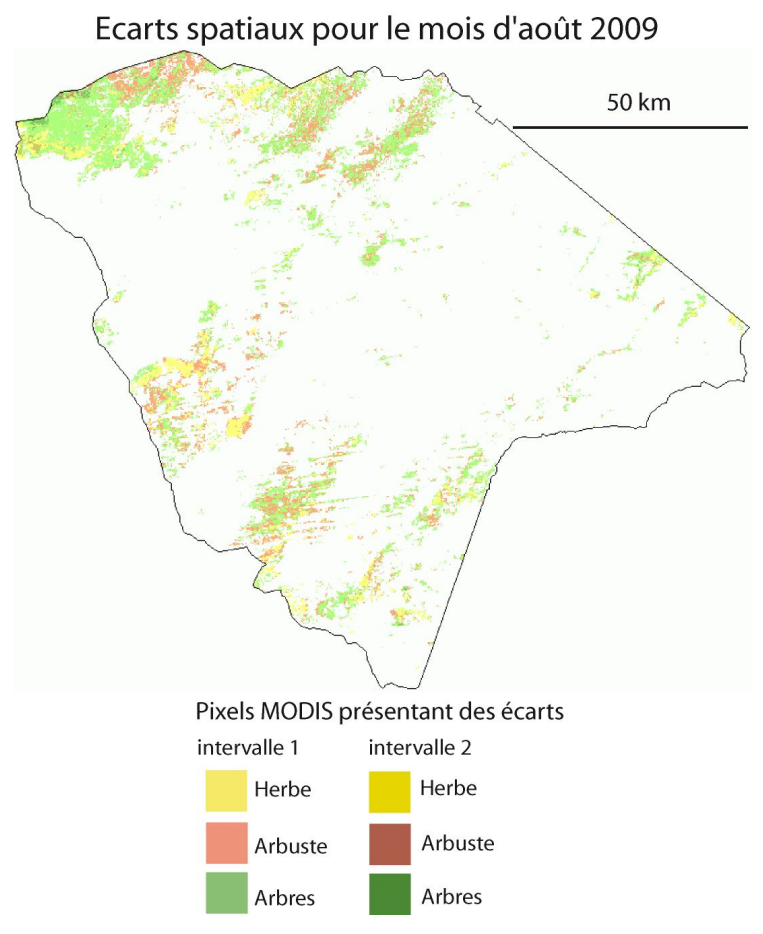

FIGURE 11 : Cartographie des pixels présentant une faible couverture végétale ("intervalle 1") ou une très faible couverture végétale ("intervalle 2") par la méthode spatiale, sur le mois d'août 2009.

figure 12, pour l'année 2009-2010, en conservant la même légende que la carte précédente.

Globalement, les zones nord et sud du parc présentent une faible couverture végétale annuelle. Comme il s'agit d'une échelle annuelle, cela veut donc dire que la végétation ne repousse pas. Plusieurs raisons peuvent être invoquées :

- Ces deux zones présentent une géologie différente que le reste du parc. II s'agit de basalte et de calcaire, tandis qu'au centre du parc se trouve du sable de Kalahari. Les végétaux peuvent moins bien y pousser.

- L'altitude est aussi différente : ces deux zones sont à $50 \mathrm{~m}$ plus basses que le reste du parc (lui-même à $1000 \mathrm{~m}$ d'altitude). II devrait donc y faire plus chaud, et peut-être changer les développements des plantes.

- Une forte présence d'animaux. Ce point est une hypothèse et reste à vérifier.

Pour résumer cette partie, nous avons mis au point des méthodes pour identifier des pixels dont la couverture végétale est anormalement faible annuellement. II s'agit bien de décrire un état faible de la couverture végétale, selon 3 modalités : une couverture végétale normale (en blanc sur la carte), une autre faible (couleurs claires) et une dernière très faible (couleurs foncées). Nous avons fait ce travail pour deux années, 20092010, comme présenté ici, et 2002-2003. Pour continuer
Ecarts spatiotemporels pour l'année 2009-2010

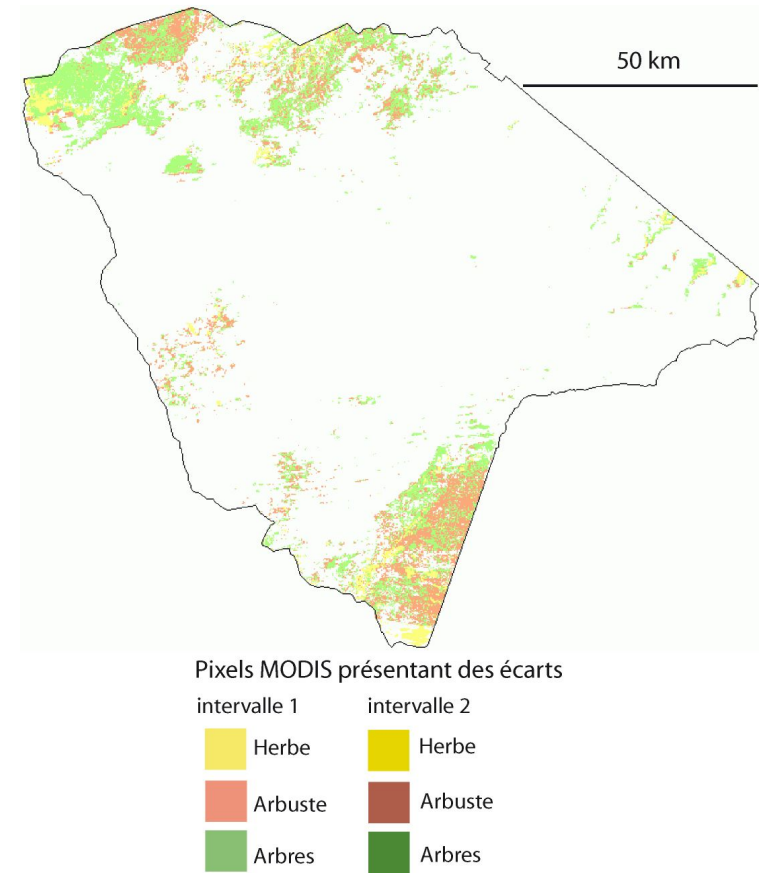

FIGURE 12 : Cartographie des pixels présentant une faible couverture végétale ("intervalle 1") ou une très faible couverture végétale ("intervalle 2") par la méthode spatio-temporelle, sur l'année 2009-2010.

nos analyses, nous conservons les résultats issus de la méthode spatio-temporelle puisqu'il s'agit de la méthode la plus aboutie et prenant en compte les aspects spatiaux et temporels.

\subsection{Evolutions des états annuels de la couverture végétale dans les pixels}

Les états annuels de la couverture végétale identifiés pour ces deux années, à travers 3 catégories, sont ensuite comparés. Cette comparaison met en évidence des évolutions de la couverture végétale en sept ans. De façon théorique, le croisement de ces 3 catégories d'état de la couverture végétale sur deux années fait apparaitre 9 catégories d'évolution de la couverture végétale (voir tableau 2). La couverture végétale peut rester stable, quel que soit l'état de celle-ci. Elle est croissante si elle passe d'un état moins faible. Au contraire, elle se dégrade si elle passe d'un état plus faible.

En pratique, les pixels du parc ne répartissent pas sur l'ensemble de ces neuf types d'évolution. Si on étudie cette répartition en prenant en entrée les pixels catégorisés en 2003 (sur 243279 pixels), on obtient le tableau 3. Sur les pixels de couverture végétale normale en 2003 (première ligne), 92\% d'entre eux ont une couverture végétale stable et $8 \%$ une couverture végétale qui s'est dégradée. II y a donc une grande stabilité de la couverture végétale normale.

Sur les pixels de couverture végétale faible en 2003 


\begin{tabular}{|l|l|l|l|}
\hline $\begin{array}{l}\text { Etats de la } \\
\text { couverture } \\
\text { végétale } \\
2003 \rightarrow 2010\end{array}$ & Normale & Faible & Très faible \\
\hline Normale & Stabilité & Dégradation & $\begin{array}{l}\text { Forte } \\
\text { dégrada- } \\
\text { tion }\end{array}$ \\
\hline Faible & Croissance & Stabilité & Dégradation \\
\hline Très faible & $\begin{array}{l}\text { Forte crois- } \\
\text { sance }\end{array}$ & Croissance & Stabilité \\
\hline
\end{tabular}

TABLE 2 : Les neuf catégories d'évolutions possibles de la couverture végétale. L'année 2003 est en ligne; l'année 2010 en colonne.

\begin{tabular}{|l|l|l|l|l|}
\hline $\begin{array}{l}\text { Etats de la } \\
\text { couverture } \\
\text { végétale } \\
2003 \rightarrow 2010\end{array}$ & Normale & Faible & $\begin{array}{l}\text { Très } \\
\text { faible }\end{array}$ & Total \\
\hline Normale & $92,24 \%$ & $7,76 \%$ & $0 \%$ & $100 \%$ \\
\hline Faible & $49,46 \%$ & $47,53 \%$ & $3,01 \%$ & $100 \%$ \\
\hline Très faible & $0 \%$ & $95,71 \%$ & $4,29 \%$ & $100 \%$ \\
\hline
\end{tabular}

TABLE 3 : Distributions des évolutions de la couverture végétale entre 2003 et 2010.

(deuxième ligne), $48 \%$ sont restés stables, $49 \%$ ont une couverture végétale en croissance et $3 \%$ qui s'est dégradée.

Enfin, sur les pixels de couverture végétale très faible en 2003 (troisième ligne), 4\% sont restés stables et 96\% présentent une croissance de la végétation. La couverture végétale s'est particulièrement développée dans ces deux catégories.

Notons également qu'il n'y a donc eu ni forte croissance ni forte dégradation de la végétation $(0 \%$ dans le tableau). Nous sommes intéressés surtout par la dégradation de la végétation dans le parc, pour lier cette problématique avec la densité de grands herbivores présents localement. Or d'après ce tableau, cette dégradation est peu étendue, puisqu'elle concerne peu de pixels : seuls $7 \%$ des pixels de couverture végétale normale en 2003 devenue faible en 2010 et $3 \%$ des pixels de couverture végétale faible en 2003 devenue très faible en 2010. Ces chiffres montrent, et c'est un résultat en lui-même, qu'à l'échelle du parc, la végétation s'est plutôt développée en sept ans. Cette conclusion coïncide avec celle établie par l'évolution décennale des indices NDVI annuels.

II importe maintenant de localiser ces évolutions, parce qu'il peut y avoir des phénomènes locaux d'évolutions. En effet, si les pixels présentant des dégradations de la couverture végétale, même peu nombreux, sont localisés dans un même lieu, alors la végétation s'est dégradée localement. Une cartographie de ces évolutions est proposée en figure 13. II s'agit de représenter les pixels chaque catégorie d'évolution de la couver- ture végétale dans le parc, par des couleurs différentes. Ici, les trois types de stabilité de la couverture végétale sont représentés en blanc, gris et noir; les deux types de dégradations en orange et rouge; les deux types de croissance en bleu et vert.

Les zones les plus étendues présentant des dégradations de la végétation sont au sud et au nord du parc. Cependant, on remarque aussi des zones de forme linéaire à l'est du parc et parallèles entre elles. Elles nous intéressent parce que nous savons, en utilisant des comptages d'animaux, des observations sur le terrain et des suivis GPS, que beaucoup de groupes d'animaux passent par des zones et les empruntent comme des routes ou des corridors, de façon linéaire. Ces zones peuvent donc être des lieux d'interaction forte entre les herbivores et la végétation.

Ces lieux d'évolution de la couverture végétale sont enfin à croiser avec les lieux de présences d'animaux, identifiés par des suivis GPS d'animaux ou des comptages d'animaux. Un croisement cartographique, qui consiste à cumuler les couches d'information, permet de voir une correspondance entre les lieux d'évolution et les passages d'animaux, et surtout ceux des buffles (en vert sur la figure 14), à l'est du parc uniquement. Cette correspondance donne une tendance, mais la corrélation statistique est encore à étudier.

\section{Conclusion}

En conclusion, ce papier décrit une approche quantitative pour décrire les évolutions des états annuels de la couverture végétale des pixels, à partir de séries temporelles d'images MODIS. Nous étudions pour cela les évolutions des valeurs NDVI mensuelles des pixels pour identifier des profils types d'évolution de la couverture végétale. Nous avons étudié les évolutions de la couverture végétale entre 2003 et 2010 en analysant deux séries temporelles d'images mensuelles au cours d'une année. Des pixels d'évolution de la couverture végétale ont ainsi été identifiés puis cartographiés pour en dégager des lieux d'évolution de la couverture végétale. Ce travail mêle des outils de télédétection, de cartographie et de statistiques.

Cette méthodologie donne des résultats concluants, ce qui signifie que les données sources, à savoir les séries temporelles d'images MODIS et l'indice NDVI, sont suffisantes pour décrire l'évolution de la couverture végétale dans cette zone d'étude.

Deux années seulement ont été finement analysées, espacées de sept ans, pour prouver la faisabilité de cette méthodologie. Cependant, maintenant, il faudrait obtenir l'ensemble des images MODIS entre 2002 et 2014 pour étudier la continuité des évolutions de la couverture végétale. II s'agira dans ce cas de vraies analyses d'évolutions, et non d'une analyse diachronique. Les profils types d'évolutions déjà mis au point ici pourront pour cela être réutilisés. L'analyse consistera alors à identifier les états annuels de la végétation. Dans ce cas, il y aura 


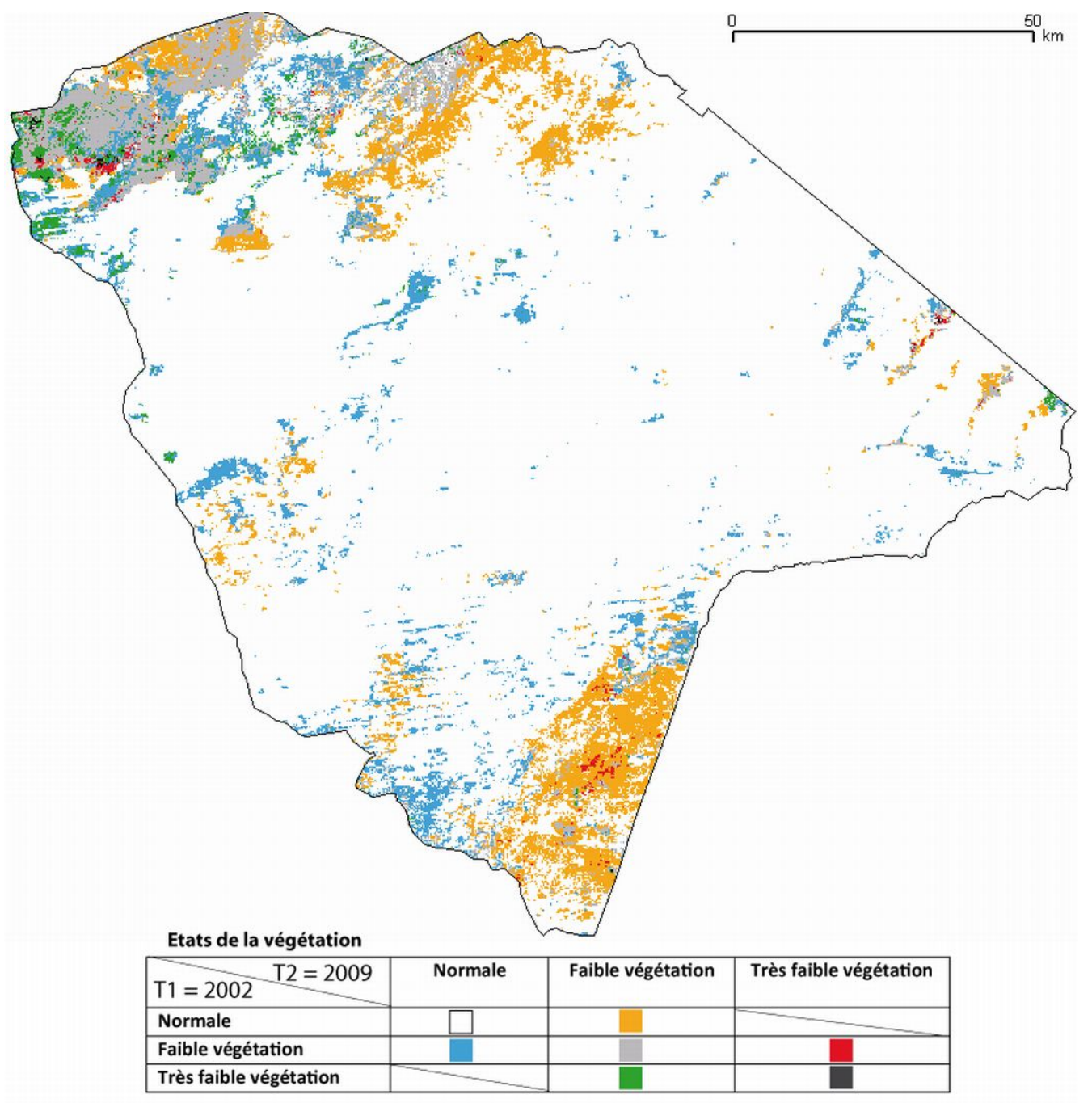

FIGURE 13 : Lieux d'évolutions de la couverture végétale en fonction des types d'évolutions entre 2003 et 2010, d'après les séries temporelles d'images MODIS. 


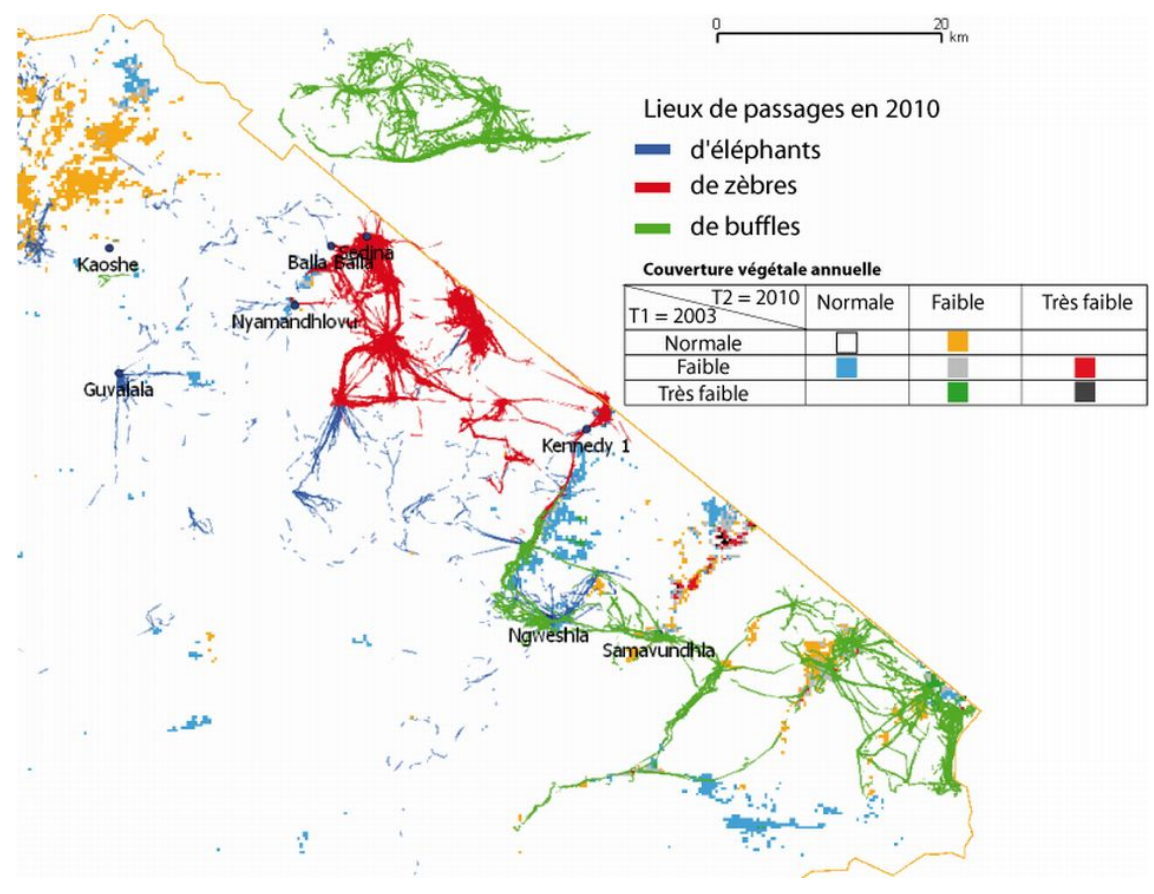

FIGURE 14 : Superposition cartographique des lieux d'évolutions de la couverture végétale entre 2003 et 2010 et des couloirs de passages d'animaux - Zoom sur l'est du parc.

aussi une grande quantité de données à analyser, ce qui posera d'autres problèmes.

Sur le parc, nos résultats sont que la végétation s'est globalement développée en sept ans, ce qui est contraire au ressenti des écologues. Néanmoins, cette évolution globale cache des disparités régionales : le sud et le nord du parc sont des zones vulnérables parce que la couverture végétale est faible et/ou dégradée en sept ans, ce qui signifie qu'elle ne se développe pas bien. II peut s'agir de caractéristiques intrinsèques aux plantes situées dans ces zones ou de facteurs externes, comme les surconsommations ou les sur passages de troupeaux d'herbivores. Très localement, des structures linéaires de $10 \mathrm{~km}$ de long peuvent être visibles sur la carte finale, à l'est du parc. Ces zones sont d'un grand intérêt puisqu'elles se situent dans une zone de fréquentation des troupeaux d'herbivores, particulièrement les troupeaux de buffles. Le travail présenté ici est donc une étape intermédiaire en vue d'étudier statistiquement les liens structurels et fonctionnels entre ces zones et les pratiques spatiales des troupeaux.

D'autres données pourraient venir en complément des images MODIS. Rappelons en effet que ces images sont de moyenne résolution spatiale et ne permettent pas d'observer des phénomènes locaux d'évolution de la couverture végétale. En particulier, sur les points d'eau, très fréquentés par les troupeaux d'animaux, on pourrait aussi utiliser d'autres images satellites ou aériennes, des photographies panoramiques prises du sol à différentes périodes ou des relevés terrain.

Enfin, notre méthodologie est facilement adaptable à d'autres problématiques et/ou d'autres zones d'études, l'idée essentielle est de retenir des pixels ayant des valeurs NDVI pour lesquelles on considère que la végétation se développe mieux ou moins bien, à différentes temporalités, puis étudier les différences entre les pixels retenus. D'autres zones d'études peuvent nécessiter de passer à un autre indicateur pour décrire la végétation, comme l'EVI s'il s'agit d'une zone tropicale humide. Même si cette adaptation est facile en théorie, ce travail est chronophage car il est fondé sur des seuils (de valeurs NDVI) et nécessite donc de se constituer une base de connaissances sur la végétation in situ.

\section{Références}

Arvor D., 2009. Etude par télédétection de la dynamique du soja et de l'impact des précipitation sur les productions au Mato Grosso (Brésil). PhD thesis, University Rennes 2, France.

Arvor D., Dubreuil V., 2006. Apport des images NDVI du satellite SPOT-végétation à l'étude de l'impact des précipitations sur les rendements de soja au Mato Grosso (Brésil). In : Proc. of Colloque "Interactions Nature-Société, analyses et modèles", 6 p, La Baule, France.

Buard E., 2013. Dynamiques des interactions espèces? espace, Mise en relation des pratiques de déplacement des populations d'herbivores et de l'évolution de l'occupation du sol dans le parc de Hwange (Zimbabwe). PhD thesis, University Paris 1, France. 
Chamaillé-Jammes S., Valeix M., Bourgarel M., Murindagomo F., Fritz H., 2009. Seasonal density estimates of common large herbivores in Hwange National Park, Zimbabwe. African Journal of Ecology, 47, 804-808.

Derrien M., Farki B., Le Gleau H., Sairouni A., 1992. Classification de la végétation sur la France à l'aide de l'AVHRR de NOAA-11. Norois, 155, 269-282.

Doll D., 2010. Apport et analyse des images satellites MODIS pour le suivi des douglassaies en Bourgogne. rapport de stage, M2 Geobiosphere, 57p.

FAO, 1998. Multiple frame agricultural surveys. Agricultural survey programs based on area frame or dual frame (area and list) sample designs. Food and Agriculture Organization of the United Nations, FAO Statistical Development Series, Vol. 2, Roma, Italy.

Ferreira L., Yoshioka H., Huete A., Sano E., 2003. Seasonal landscape and spectral vegetation index dynamics in the Brazilian Cerrado: An analysis within the Large-Scale Biosphere-Atmosphere Experiment in Amazonia(LBA). Remote Sensing of Environment, 87(4), 534-550.

Frontier S., 1977. Réflexion pour une théorie des ecosystems. Bulletin of Ecology, 8(4), 445-464.

Ganzin N., Crosmary W., Fritz H., 2008. A simplified vegetation map of Hwange National Park and surrounding areas using Landsat ETM+ satellite imagery. Working document, June 2008, 26 p.

Hubert-Moy L., Cotonnec A., Le Du L., Chardin A., Perez P., 2001. A comparison of classification procedures of remotely sensed data applied on different landscape units. Remote Sensing of Environment, 75 (2), 174-187.

Huete A., Didan K., Miura T., Rodriguez E. P., Gao X., Ferreira L. G., 2002. Overview of the radiometric and biophysical performance of the MODIS vegetation indices, Remote Sensing of Environment, 83(1-2), 195-213.

Jacquin A., 2010. Dynamique de la végétation des savanes en lien avec l'usage des feux à Madagascar Analyse par série temporelle d'images de télédétection. PhD thesis, University de Toulouse, France.

Lindeman R.L., 1942. The trophic-dynamic aspect of ecology. Ecology, 23, 399-418.

Lovegrove B. G., Haines L. M., 2004. The Evolution of Placental Mammal Body Sizes : Evolutionary History, Form, and Function. Oecologia, 2004, 138, 13-27.
Marteau R., 2006. Sensibilité de la relation précipitations - NDVI (1981 - 1999) aux états de surfaces continentaux sur l'ouest du plateau sud-africain. MSc thesis CEPS. University of Burgundy, France.

Meneses-Tovar C.L., 2011. NDVI as indicator of degradation. Unasylva, 238 (62), 39-46.

Najib D., 2010. Exploitation des images satellitaires MODIS-TERRA pour la caractérisation des états de surface. MSc thesis. University of Laval, Québec City, Canada.

Owen-Smith N., 1988. Megaherbivores : the influence of very large body size on ecology. Cambridge University press, Cambridge, United Kingdom.

Paegelow M., Maestripieri N., Saez Villalobos N., Toro Balbontin D.,2012. Détection des plantations forestières par imagerie satellite MODIS dans le Sud du Chili. M@ppemonde, n`106.

Rackham O., 2008. Ancient woodlands : modern threats. New Phytologist, 180(3), 571 ?586.

Richard Y., Poccard I., 1998. A statistical study of the NDVI sensitivity to seasonal and interannual rainfall variations in southern Africa. International Journal of Remote Sensing, 19 (15), 2907-2920.

Rogers A., 1993. A woody vegetation survey of Hwange National Park. Available at : www.getcited.org/pub/103270828 [Accessed March 2, 2011].

Rouse J., Haas R., Schell J., Deering D., Harlan J., 1974. Monitoring the vernal advancement of retrogradation of natural vegetation, NASA/GSFC, Type III, Final Report, Greenbelt, $371 \mathrm{p}$.

UICN (Union Internationale pour la Conservation de la Nature), 2008. 2008 IUCN Red List of Threatened Species.

Valeix M., Fritz H., Sabatier R., Murindagomo F., Cumming D., Duncan P., 2011. Elephant-induced structural changes in the vegetation and habitat selection by large herbivores in an African savanna. Biological Conservation. 144, 902-9012.

Van Aarde R., Jackson T., 2006. The big picture. Africa Geographic, April 2006, 76-79. 